\section{Germ cell aneuploidy in zebrafish with mutations in the mitotic checkpoint gene mps1}

\author{
Kenneth D. Poss, ${ }^{1,2,4,7,10}$ Alex Nechiporuk, ${ }^{1,2,4,8}$ \\ Keith F. Stringer, ${ }^{1,2,4,5,9}$ Charles Lee, ${ }^{3,6}$ \\ and Mark T. Keating ${ }^{1,2,4}$
}

\begin{abstract}
${ }^{1}$ Howard Hughes Medical Institute, ${ }^{2}$ Department of Cell Biology and ${ }^{3}$ Department of Pathology, Harvard Medical School, Boston, Massachusetts 02115, USA; ${ }^{4}$ Department of Cardiology and ${ }^{5}$ Department of Pathology, Children's Hospital, Boston, Massachusetts 02115, USA; ${ }^{6}$ Department of Pathology, Brigham and Women's Hospital, Boston, Massachusetts 02115, USA; ${ }^{7}$ Department of Cell Biology, Duke University Medical Center, Durham,

North Carolina 27710, USA
\end{abstract}

Aneuploidy, resulting from chromosome missegregation during meiosis, is a major cause of human infertility and birth defects. However, its molecular basis remains incompletely understood. Here we have identified a spectrum of chromosome anomalies in embryos of zebrafish homozygous for a hypomorphic mutation in Mps1, a kinase required for the mitotic checkpoint. These aneuploidies are caused by meiotic error and result in severe developmental defects. Our results reveal Mps1 as a critical regulator of chromosome number in zebrafish, and demonstrate how slight genetic perturbation of a mitotic checkpoint factor can dramatically reduce the fidelity of chromosome segregation during vertebrate meiosis.

Received December 30, 2003; revised version accepted May 11, 2004.

More than $20 \%$ of human conceptions have chromosome disorders. Strikingly, $\sim 50 \%$ of spontaneous abortions before 15 wk gestation are caused by aneuploidy, the loss or gain of one or more individual chromosomes. Also, aneuploidy represents the most common genetic defect at birth, affecting three in every 1000 newborns. Aneuploidy is predominantly maternal and increases dramatically in incidence with maternal age (Nicolaidis and Petersen 1998; Hassold and Hunt 2001).

Work from yeast and invertebrate model systems has generated the idea that meiotic chromosome segregation errors are due in part to deficiencies in mitotic checkpoint activity. The mitotic checkpoint monitors the integrity of the mitotic spindle and detects the presence of

[Keywords: Zebrafish; aneuploidy; meiosis; spindle checkpoint; Mps1; nondisjunction]

Present addresses: ${ }^{8}$ Department of Biological Structure, University of Washington, Seattle, WA 98195, USA; ${ }^{9}$ Department of Pathology, Cincinnati Children's Hospital Medical Center, Cincinnati, OH 45229, USA. ${ }^{10}$ Corresponding author.

E-MAIL k.poss@cellbio.duke.edu; FAX (919) 684-5481.

Article and publication are at http://www.genesdev.org/cgi/doi/10.1101/ gad.1182604 misaligned chromosomes in dividing somatic cells (Cleveland et al. 2003). If segregation errors occur, it facilitates their correction by arresting cells in metaphase through inhibition of the anaphase-promoting complex, limiting the generation of aneuploid cells. Several genes have been shown to be essential for activation of the mitotic checkpoint. In the budding yeast, Saccharomyces cerevisiae, these include MAD1-3, BUB1-3, and MPS1. Notably, during meiosis I, loss-of-function mutations in S. cerevisiae MPS1, MAD1 (a substrate of Mps1), and MAD2 cause nondisjunction events (Shonn et al. 2000; Straight et al. 2000). In Caenorhabditis elegans, RNAi knockdown of $m d f-1$ or $m d f-2$, orthologs of $M A D 1$ or $M A D 2$, leads to meiotic error (Kitagawa and Rose 1999|. Among vertebrates, recent gene targeting experiments using mice revealed an essential role for mitotic checkpoint genes in chromosome segregation during early mammalian embryogenesis. However, because of the embryonic lethality caused by homozygous null mutations in these genes, the roles for mitotic checkpoint genes during meiosis could not be assessed (Dobles et al. 2000; Kalitsis et al. 2000; Babu et al. 2003). Thus, there is currently no vertebrate model in which the mitotic checkpoint is disrupted in germ cells.

The dual specificity kinase Mps1 is essential for activation of the mitotic checkpoint in budding and fission yeasts, and in amphibian, zebrafish, and human cells (Weiss and Winey 1996; He et al. 1998; Abrieu et al. 2001; Poss et al. 2002a; Stucke et al. 2002). In vertebrates, a mechanism for Mps1 was recently proposed in which it is required upstream in the mitotic checkpoint signaling pathway to regulate the levels of mitotic checkpoint proteins such as Mad1 and Mad2 on kinetochores (Abrieu et al. 2001). In this model, Mps1 activity defines a precise level of kinetochore-localized checkpoint proteins to facilitate progression of error-free mitosis. Recent siRNA and antibody knockdown experiments in human cells have confirmed that Mps1 is required to prevent chromosome segregation errors during normal mitotic progression (Fisk et al. 2003; Liu et al. 2003).

Recently, we identified a temperature-sensitive mutation in zebrafish called mps1 (mps $\left.1^{z p 1}\right)$ during a mutagenesis screen for caudal fin regeneration defects (Poss et al. 2002a). In this strain, a lysine replaces a conserved isoleucine at amino acid 843 within the large, carboxyl kinase domain. Homozygous $m p s 1^{z p 1}$ embryos derived from heterozygous parents are inviable when raised at the restrictive temperature of $33^{\circ} \mathrm{C}$. In addition, both fin and heart regeneration fail in adult mutants given a temporary $33^{\circ} \mathrm{C}$ incubation, deficiencies that are associated with impaired cellular proliferation (Poss et al. 2002a,b). These proliferative defects were attributed to reductions in mitotic checkpoint activity displayed by $m p s 1^{z p 1}$ cells at the restrictive temperature. In contrast, when raised at the permissive temperature $\left(25^{\circ} \mathrm{C}-28^{\circ} \mathrm{C}\right)$, a high percentage of homozygous mps $1^{z p 1}$ animals survive to adulthood. Importantly, adult $m p s 1^{z p 1}$ homozygotes are indistinguishable from wild-type siblings in gross behavior, breeding, and life span.

In this study, we report that mps1 is concentrated in male and female zebrafish reproductive tissues, and that the $m p s 1^{z p 1}$ mutation reduces mitotic checkpoint activ- 
ity and leads to chromosome anomalies in male germ cells. Moreover, in spite of the normal appearance of sexually mature $m p s 1^{z p 1}$ males and females, their progeny display a variety of developmental defects attributable to severe aneuploidies. Our results demonstrate that Mps1 is a crucial factor for maintaining chromosome number in zebrafish, and suggest that vertebrate germ cells are exquisitely sensitive to disruptions in the activity of mitotic checkpoint genes.

\section{Results and Discussion}

Dysmorphology in embryos from mps $1^{\mathrm{zp} 1}$ parents

Only $\sim 1 \%$ of progeny from adult $m p s 1^{z p 1}$ homozygous matings survived to adulthood at the permissive temperature. Initial defects were observed during gastrulation (data not shown), leading to severe, heterogeneous patterning problems by $28 \mathrm{~h}$ postfertilization (hpf). The predominant deformities at $28 \mathrm{hpf}$ included head degeneration, antero-posterior truncations, and/or dysmorphic somites. A small percentage of offspring displayed a complete lack of morphogenesis, or conspicuous patterning defects such as duplications (Fig. 1A). By outcrossing $m p s 1^{z p 1}$ males and females to wild-type zebrafish, we found that the $m p s 1^{z p 1}$ mutation had both paternal and maternal effects. In paternal mps $1^{z p 1}$ crosses, $\sim 26 \%$ of embryos were dysmorphic at $28 \mathrm{hpf}$ (average percent dysmorphology per mating, $n=11$ matings; 1369 total embryos), whereas $\sim 46 \%$ of embryos from maternal mps $1^{z p 1}$ crosses showed morbidity ( $n=10$ matings; 860 embryos). The paternal and maternal effects of the $m p s 1^{z p 1}$ lesion appeared additive, as $\sim 71 \%$ of embryos from homozygous $m p s 1^{z p 1}$ matings displayed severe defects $(n=14$ matings; 685 embryos; Fig. 1B). Statistical analyses indicated significant differences between the frequency of dysmorphic progeny from $m p s 1^{z p 1}$ fathers versus that from $m p s 1^{z p 1}$ mothers, and between families with only one $m p s 1^{z p 1}$ parent versus families with two $m p s 1^{z p 1}$ parents.

\section{mps 1 is localized to male and female germ cells}

We suspected that the most likely basis for this unusual mechanism of inheritance was an essential function for Mps1 in zebrafish germ cells. Therefore, we first examined whether mps1 is preferentially expressed in reproductive tissues. Indeed, mps $1 \mathrm{mRNA}$ was expressed in ovary and testis at levels much higher than in any of the somatic tissues tested (Fig. 2A). Zebrafish ovaries consist of a fairly synchronous population of larger oocytes (a clutch), and a more heterogeneous population of smaller oocytes, from which the clutch is recruited (Wallace and Selman 1981; Uchida et al. 2002). Using in situ hybridization, we found an abundance of mps1 mRNA in smaller, immature perinucleolar and previtellogenic oocytes with intact germinal vesicles, but much lower levels in larger, more mature vitellogenic oocytes (Fig. 2B). Zebrafish testes are composed of cyst-like structures, with spermatogonial stem cells, spermatocytes, and sperm distinguishable by nuclear size and morphology (Grier 1981; Uchida et al. 2002). mps1 mRNA was strongly expressed in primary (pre-meiosis I) and secondary (pre-meiosis II) spermatocytes (Fig. 2C), but we detected little or none in mature spermatozoa. Thus, mps 1 mRNA is concentrated in immature germ cells that have not completed meiosis.
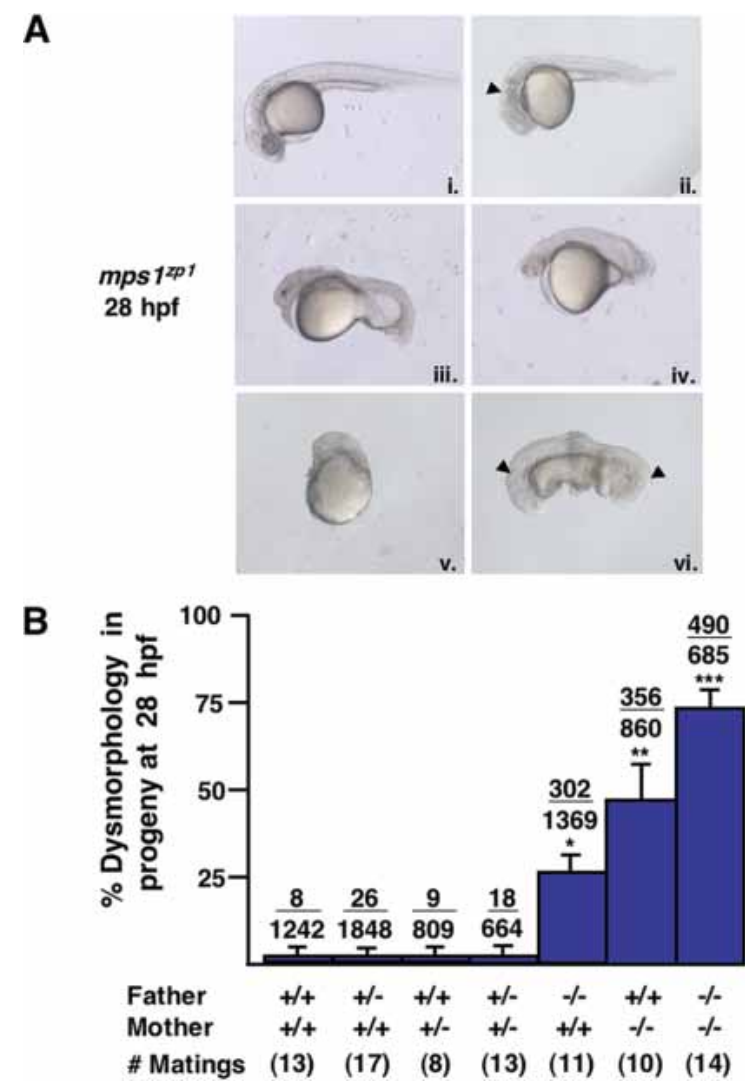

Figure 1. Zebrafish homozygous for $m p s 1^{z p 1}$ generate dysmorphic offspring. (A) One-quarter to one-third of embryos from $m p s 1^{z^{p 1}}$ parents appeared normal at $28 \mathrm{hpf}$ (panel i). A significant proportion of embryos displayed head degeneration (panel ii, arrowhead), whereas others displayed severe defects in head, somite, and trunk development (panels iii,iv). In a small percentage of embryos, no head or trunk was distinguishable (panel $v$ ), or spectacular patterning defects were noted (panel vi). This embryo has two joined head and trunk structures in the absence of posterior structures (arrowheads point to otic vesicles). (B) Frequency of dysmorphic progeny from different combinations of wild-type $(+/+), m p s 1^{z p 1}$ heterozygous $(+\mid-)$, and $m p s 1^{z p 1}$ homozygous $(-/-)$ parents. Data are shown as mean \pm S.E.M. Student's $t$-test, $\left(^{\star}\right) p \ll 0.001$ versus two $+/+$ parents. $\left({ }^{\star *}\right) p \ll 0.001$ versus two $+/+$ parents, $p<0.05$ versus $-/-$ father and $+/+$ mother. $\left(^{\star \star \star}\right) p \ll 0.001$ versus two $+/+$ parents, $p \ll 0.001$ versus $-/$ - father and $+/+$ mother, $p<0.05$ versus $+/+$ father and $-/-$ mother. Numbers above the error bars represent the total number of dysmorphic embryos among the crosses, divided by the total number of embryos analyzed. The majority of $m p s 1^{z^{1} 1}$ animals in these experiments were 7-10 mo of age. In our facilities, zebrafish retain fecundity from 4 to 15 mo of age, and live $2-3$ yr.

\section{The mps $1^{\mathrm{zp} 1}$ mutation disrupts mitotic checkpoint activity and alters chromosome content in male germ cells}

Because of the high frequency and variability of defects in embryos from $m p s 1^{z p 1}$ parents, we predicted that the mps $1^{z p 1}$ mutation caused chromosome disorders in embryos. Precisely how it caused these disorders was unclear, however, because multiple functions have been assigned to Mps 1 in eukaryotes. In growing Saccharomyces cerevisiae, Mpsl regulates duplication of the spindle pole body (the analogous structure to the vertebrate centrosome) and is essential for the mitotic checkpoint (Winey et al. 1991; Weiss and Winey 1996). Analogous roles have been identified in vertebrate species, although 


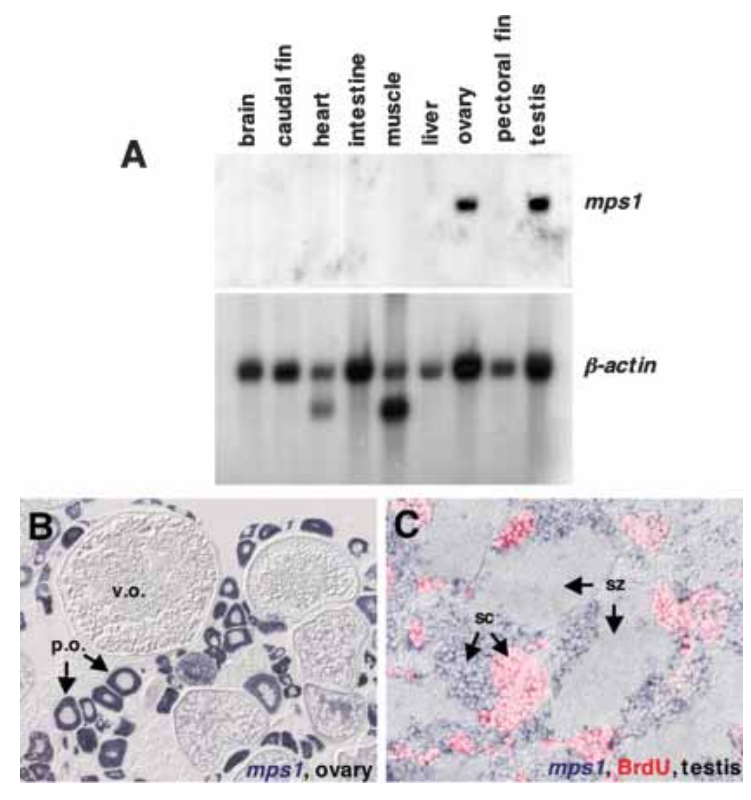

Figure 2. mps1 is localized to immature zebrafish germ cells. $(A)$ Northern analysis of mps1 expression using several adult tissues. RNA samples from testis and ovary, although slightly overloaded on this blot, are the only tissues that demonstrate mps1 expression. (B) In situ hybridization of ovarian tissue section detects strong $m p s 1$ expression (violet stain) in small, immature perinucleolar oocytes (p.o.). Mature vitellogenic oocytes (v.o.) show little or no mps1 expression. $(C)$ In testis, mps1 mRNA is localized to primary and secondary spermatocytes (sc), with little or no expression in spermatozoa (sz). To confirm the identity of mps1-positive cells, animals were injected with BrdU $5 \mathrm{~h}$ prior to tissue processing. In $5 \mathrm{~h}$, BrdUpositive cells (pink) result from mitotic division of spermatogonial stem cells to yield primary spermatocytes, or mitotic division plus one meiotic division to yield secondary spermatocytes. All BrdUpositive cells were also mps1-positive; mature spermatozoa were BrdU- and mps1-negative.

in vertebrates, Mps1 is required for normal, unperturbed mitotic progression (Abrieu et al. 2001; Fisk and Winey 2001; Poss et al. 2002a; Stucke et al. 2002; Fisk et al. 2003; Liu et al. 2003). Thus, the mps $1^{z p 1}$ mutation might cause chromosome segregration errors through one or both of two mechanisms: (1) failure of centrosome duplication, leading to monopolar spindles and multinucleated gametes, or (2) failure in mitotic checkpoint activity, causing nondisjunction (i.e., sporadic monosomies and trisomies). To address this issue, we assessed mitotic spindles and mitotic checkpoint activity in wild-type and $m p s 1^{z p 1}$ tissues. Because assays of mitotic checkpoint activity and chromosome content can be readily adapted to assess male germ cells, we used male zebrafish for these studies.

To assess gross defects in spindle formation, we microscopically examined meiotic cells in tissue sections for centrosome number and spindle integrity. We saw no evidence of centrosome deficiency or monopolar spindles in testes of $m p s 1^{z p 1}$ zebrafish maintained at $25^{\circ} \mathrm{C}$ or incubated for $12 \mathrm{~h}$ at $33^{\circ} \mathrm{C}$, the restrictive temperature for the $m p s 1^{z p 1}$ mutation during embryogenesis and regeneration (Fig. 3A,B). To investigate mitotic checkpoint activity in zebrafish germ cells, we assessed the response of $m p s 1^{z p 1}$ animals and wild-type and heterozygous clutchmates to incubation in nocodazole, an agent that disrupts mitotic spindles and causes an arrest in metaphase that is dependent on mitotic checkpoint activity. Heterozygous and wild-type zebrafish treated with nocodazole for $12 \mathrm{~h}$ at $25^{\circ} \mathrm{C}$ or $33^{\circ} \mathrm{C}$ showed a dramatic increase in meiotic cells (as assessed by nuclei that were positive for phosphorylated histone-3 [H3P] immunoreactivity). We noted a similar increase in meiotic cells in $m p s 1^{z p 1}$ zebrafish treated at $25^{\circ} \mathrm{C}$ (data not shown). In contrast, nocodazole treatment caused no increase in the number of meiotic cells in $m p s 1^{z p 1}$ zebrafish treated at $33^{\circ} \mathrm{C}$, clearly revealing a deficiency in germ-cell mitotic checkpoint activity (Fig. 3C).

We infer from this finding that, at $25^{\circ} \mathrm{C}$, the hypomorphic effects of the mps $1^{z p 1}$ mutation on mitotic checkpoint activity manifest in mitosis as reduced survival to adulthood $(\sim 50 \%$; Poss et al. 2002a), and in meiosis as occasional errors in chromosome segregation. When the
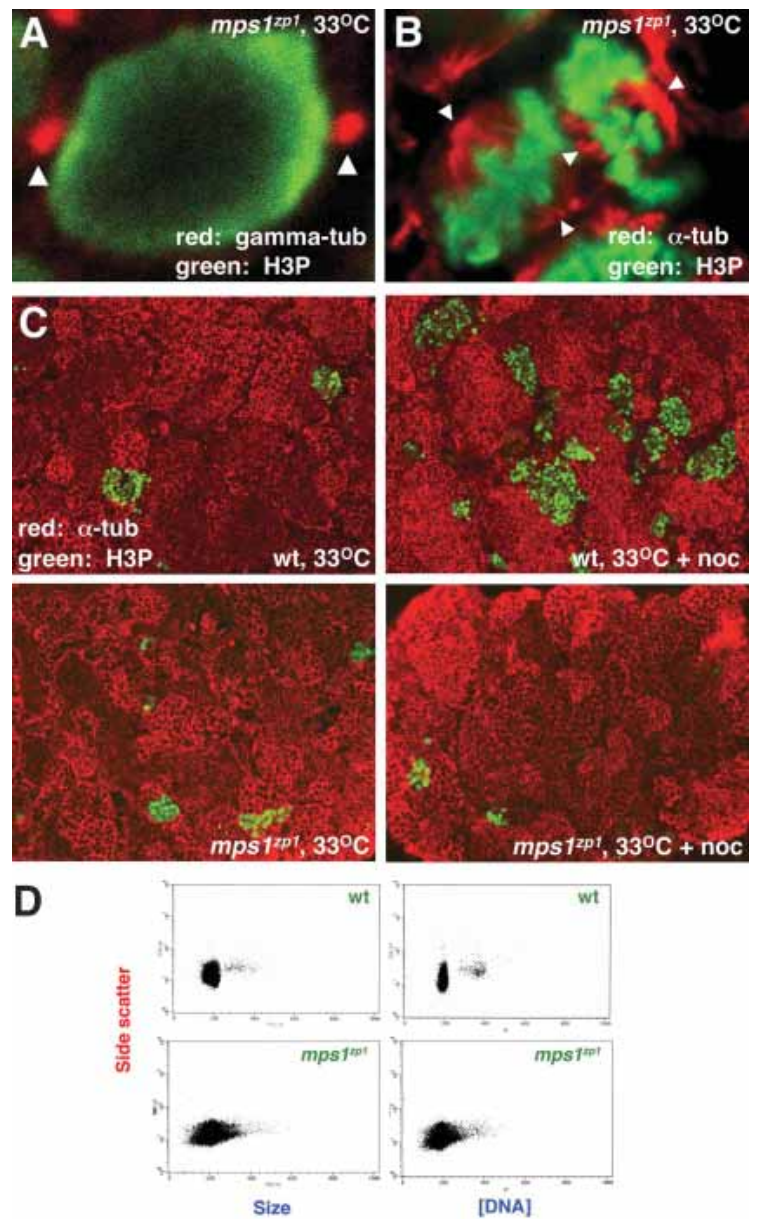

Figure 3. Mitotic checkpoint deficits and altered chromosome content in germ cells of male $m p s 1^{1 p 1}$ zebrafish. $(A)$ Confocal image of a meiotic cell from the testis of an $m p s 1^{z p 1}$ male treated for $12 \mathrm{~h}$ at $33^{\circ} \mathrm{C}$, stained for $\gamma$-tubulin (red) and H3P (green). Arrowheads indicate two centrosomes. (B) Confocal image of two meiotic cells from the testis of an $m p s 1^{1 p 1}$ male treated for $12 \mathrm{~h}$ at $33^{\circ} \mathrm{C}$, stained for $\alpha$-tubulin (red) and H3P (green). Each mitotic spindle has two clear poles (arrowheads). (C) Sections of testes from wild-type $(t o p)$ and mps1 $1^{21}$ (bottom) zebrafish treated with (left) or without (right) nocodazole for $12 \mathrm{~h}$ at $33^{\circ} \mathrm{C}$, stained for $\alpha$-tubulin (red) and $\mathrm{H} 3 \mathrm{P}$ (green). Nocodazole treatment activates the mitotic checkpoint and induces arrest in wild-type animals, indicated by increases in meiotic cells, but has no effect in mps $1^{z p 1}$ clutchmates. $(D)$ FACS analysis of sperm from representative wild-type (top) and $\mathrm{mps}^{11^{1 p 1}}$ (bottom) males. $m p s 1^{1 p 1}$ sperm displayed a wide range of size and DNA content (as assessed by propidium iodide fluorescence). 
strength of the mutation is increased by raising water temperature to $33^{\circ} \mathrm{C}$, more severe phenotypes prevail in both mitosis (embryonic lethality, regenerative failure) and meiosis (failure to arrest in the absence of mitotic spindles). That no gross defects in centrosome number or spindle morphology could be detected in the background of the hypomorphic mps $1^{z p 1}$ allele is consistent with recent findings by Fisk et al. (2003), which indicated that the mitotic checkpoint function is more sensitive to a reduction in Mps1 activity than the centrosome duplication function. However, it is possible that more sensitive tests of synchronized $m p s 1^{z p 1}$ cells in culture could reveal defects in centrosome duplication. To examine whether chromosomal nondisjunction indeed occurs in germ cells of $m p s 1^{z p 1}$ zebrafish at $25^{\circ} \mathrm{C}$, we used flow cytometry to analyze the size and chromosome content of large numbers of sperm collected from $m p s 1^{z p 1}$ and wild-type males. $m p s 1^{z p 1}$ sperm displayed a much wider range of size and DNA content than wild-type sperm (Fig. 3D), indicative of the accumulation of chromosomal gains and losses. Thus, the $m p s 1^{z p 1}$ mutation reduces mitotic checkpoint activity in male meiotic cells, a disruption that is associated with anomalies in chromosome number in $m p s 1^{z p 1}$ male gametes and is likely to cause aneuploidy in $m p s 1^{z p 1}$ progeny.

\section{A high frequency of aneuploidy in progeny of mps $1^{\text {zp1 }}$ zebrafish}

To visualize and confirm aneuploidy in $m p s 1^{z p 1} \mathrm{em}-$ bryos, we used fluorescent in situ hybridization (FISH). Wild-type embryos, as well as normal-appearing embryos from $m p s 1^{z p 1}$ matings, were disomic for linkage group 9 (LG9) and LG17, based on FISH with probes specific for near-centromeric loci of these chromosomes. Interestingly, FISH detected monosomies, trisomies, and even tetrasomies in several grossly dysmorphic embryos from crosses of $m p s 1^{z p 1}$ parents maintained at $25^{\circ} \mathrm{C}$ (Table 1; Fig. 4A). These findings demonstrate that the heterogeneous morphological defects that we observed in $m p s 1^{z p 1}$ progeny were caused by defects in chromosome number.
To determine the nature and frequency of nondisjunction events in $m p s 1^{z p 1}$ germ cells, we genotyped 470 dysmorphic, $28 \mathrm{hpf}$ embryos from (mps $1^{z p 1} \times$ wild-type) crosses for centromere-linked, polymorphic markers representing five chromosomes (Fig. 4B). Genotypic analysis allowed us to unequivocally define chromosomal inheritance in these affected $m p s 1^{z p 1} /+$ embryos. Only one of these $m p s 1^{z p 1} /+$ embryos failed to inherit precisely one moiety of each tested chromosome from a wild-type parent (1813 sets of chromosomes analyzed; $0.06 \%)$, indicating that the wild-type zebrafish germ line normally has a very low rate of nondisjunction. However, within dysmorphic embryos collected from several paternal mps $1^{z p 1}$ crosses (representing $22 \%$ of total embryos), an average of $7.5 \%$ of the analyzed chromosome sets per mating lacked the chromosome of paternal origin. Another $8.5 \%$ of chromosome sets showed inheritance of two paternally-derived homologous chromosomes (Fig. 4B). Within dysmorphic embryos from maternal mps $1^{z p 1}$ crosses (representing $47 \%$ of total embryos), an average of $14.4 \%$ of the analyzed chromosome sets per mating lacked the chromosome of maternal origin, while another $20.1 \%$ showed inheritance of two maternally-derived homologous chromosomes. Nondisjunction frequencies were similar for each of five chromosomes tested in our embryo panel, and each aneuploid embryo in our panel had a unique five-chromosome enumeration signature (data not shown). In summary, dysmorphic embryos from $m p s 1^{z p 1}$ fathers had only an $84 \%$ chance of inheriting one paternal chromosome for each chromosome set, while those from $m p s 1^{z p 1}$ mothers had only a $65 \%$ chance of inheriting one maternal chromosome for each set. Thus, both chromosomal gains and losses occurred in extremely high frequencies within $m p s 1^{z p 1}$ germ lines.

\section{Conclusions}

We conclude from our experiments that a reduction in mitotic checkpoint activity via a hypomorphic mps1 mutation, although not severe enough to completely disrupt development or physiology, is detrimental to mei-

Table 1. Chromosome number (CN) analysis by two-color FISH of cells isolated from wild-type and mps1 ${ }^{\text {zp1 }}$ zebrafish embryos

\begin{tabular}{|c|c|c|c|c|c|c|c|}
\hline \multirow[b]{2}{*}{ Genotype } & \multicolumn{6}{|c|}{ CN of individual cells for LG9, LG17 } & \multirow{2}{*}{$\frac{\text { LG9, LG17 }}{\text { Average CN }}$} \\
\hline & No. of cells & Disomy & Monosomy & Trisomy & Tetrasomy & Other & \\
\hline \multirow[t]{6}{*}{ WT (6) } & 15 & 15,15 & 0,0 & 0,0 & 0,0 & 0,0 & $2.0,2.0$ \\
\hline & 15 & 15,15 & 0,0 & 0,0 & 0,0 & 0,0 & $2.0,2.0$ \\
\hline & 15 & 15,15 & 0,0 & 0,0 & 0,0 & 0,0 & $2.0,2.0$ \\
\hline & 15 & 15,13 & 0,2 & 0,0 & 0,0 & 0,0 & $2.0,1.9$ \\
\hline & 15 & 15,14 & 0,1 & 0,0 & 0,0 & 0,0 & $2.0,1.9$ \\
\hline & 15 & 15,14 & 0,1 & 0,0 & 0,0 & 0,0 & $2.0,1.9$ \\
\hline \multirow[t]{6}{*}{$m p s 1^{z p 1}(6)$} & 15 & 12,2 & 1,12 & 1,0 & 0,0 & $0,1^{\mathrm{a}}$ & $2.1,1.1$ \\
\hline & 15 & 0,12 & 0,2 & 15,1 & 0,0 & 0,0 & $3.0,1.9$ \\
\hline & 12 & 3,9 & 0,1 & 8,2 & 1,0 & 0,0 & $2.8,2.1$ \\
\hline & 15 & 8,0 & 3,0 & 2,6 & 0,6 & $2^{\mathrm{a}}, 3^{\mathrm{b}}$ & $1.7,3.8$ \\
\hline & 15 & 15,15 & 0,0 & 0,0 & 0,0 & 0,0 & $2.0,2.0$ \\
\hline & 15 & 14,14 & 1,1 & 0,0 & 0,0 & 0,0 & $1.9,1.9$ \\
\hline
\end{tabular}

All parents were maintained at $25^{\circ} \mathrm{C}$.

a'Zero chromosomes.

${ }^{\mathrm{b}}$ Five chromosomes. 


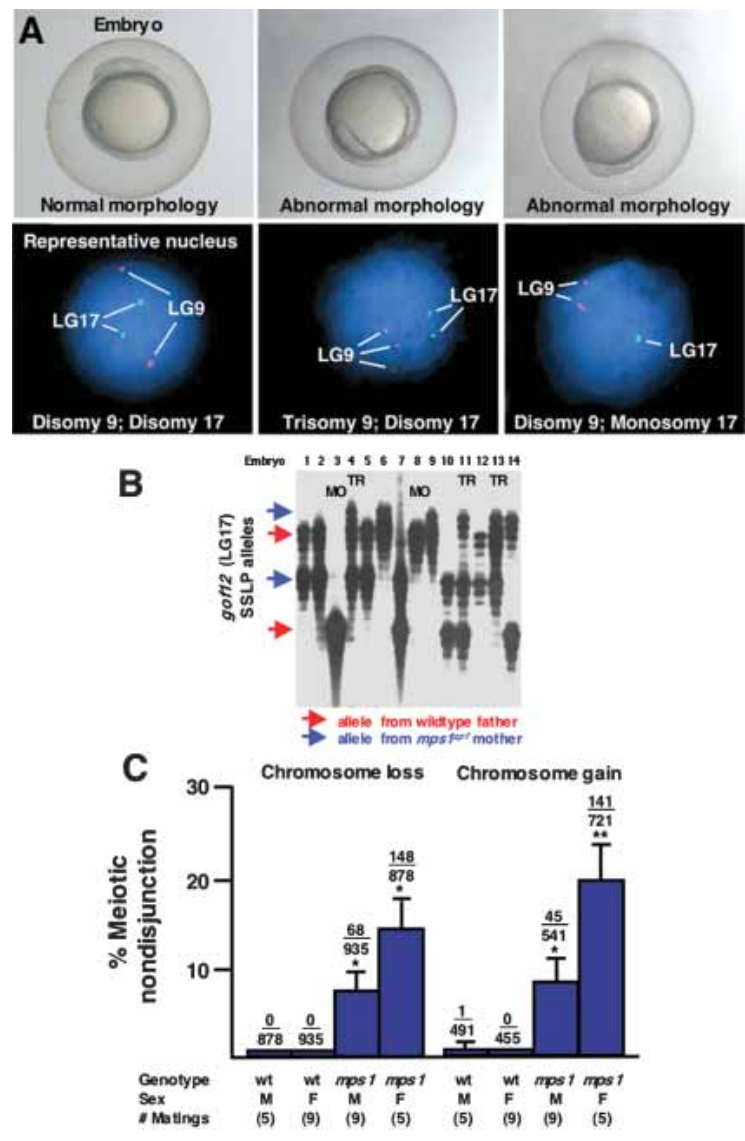

Figure 4. A high frequency of aneuploidy in $m p s 1^{2 p 1}$ germ lines. $(A)$ Eight-somite stage embryos (top), and representative FISH analyses (bottom) indicating one DAPI-stained nucleus for linkage groups 9 (red probe) and 17 (green). (Left) Wild-type embryo with elongated body, tapered tail, and normal head structure, is disomic for both linkage groups. (Center) mps $1^{z p 1}$ embryo with degenerated head and malformed trunk and tail, is trisomic for LG9. (Right) $m p s 1^{z p 1} \mathrm{em}$ bryo with blunted head and tail is monosomic for LG17. (B) Autoradiograph indicating genotypes for genetic marker gof12 on LG17, from dysmorphic progeny of a representative wild-type male $\times$ $m p s 1^{z p 1}$ female cross. Each lane represents one embryo. The two parents of this family displayed four differently sized gof12 PCR products; red arrows indicate two alleles from the wild-type father, whereas blue arrows indicate two alleles from the mps $1^{z p 1}$ mother. Note two embryos with maternal LG17 monosomy (MO) and three with maternal LG17 trisomy (TR). (C) Meiotic nondisjunction rates of wild-type and $m p s 1^{z p 1}$ fathers and mothers in wild-type $\times m p s 1^{z p 1}$ crosses. Data were compiled from analysis of 273 dysmorphic embryos from $m p s 1^{z p 1}$ fathers, and 197 dysmorphic embryos from mps $1^{z p 1}$ mothers. The numbers above the error bars represent the total number of losses (monosomy) or gains (trisomy) among five chromosomes analyzed, divided by the total number of chromosomes with informative polymorphisms. Student's $t$-test, $\left({ }^{*}\right)$ $p \ll 0.001$ versus wild-type male or female. $\left(^{* \star}\right) p \ll 0.001$ versus wild-type male or female, $p<0.05$ versus $m p s 1^{z p 1}$ father. All parents used for FISH and genotyping analyses were maintained at $25^{\circ} \mathrm{C}$.

otic chromosome segregation in both male and female zebrafish. A wide spectrum of aneuploid genotypes and developmental defects is generated by $m p s 1^{z p 1}$ zebrafish.

Interestingly, Mps1 is expressed in most rapidly cycling cells, and regeneration experiments indicate that it is required for rapid mitotic events (Poss et al. 2002a,b). Here, we have found that Mps1 is continually maintained at high levels in zebrafish germ cells undergoing meiosis, cells that appear to be even more sensitive to a reduction in Mps1 function. We suspect that this maintenance reflects a prominent role in regulating the segregation of homologous chromosomes during meiosis I, events that are distinct from mitotic chromosome segregation. That meiosis I is especially sensitive to reduced Mps1 function is supported by the fact that we detected alleles from both sister homologs, chromosomes that normally segregate during meiosis I, of $m p s 1^{z p 1}$ parents in trisomic progeny of $m p s 1^{z p 1} \times$ wild-type crosses. Because yeast meiosis is so severely affected by MPS1 mutations compared with MAD1 or MAD2 mutations, it has been proposed that Mps1 may have yet an additional function in facilitating chromosome segregation during meiosis that is separate from centrosome duplication and activation of the mitotic checkpoint through Mad2 (Straight et al. 2000; Winey and Huneycutt 2002). It is possible that such a mechanism is also mildly affected in $m p s 1^{z p 1}$ zebrafish and contributes to meiotic nondisjunction.

What molecular events cause aneuploidy in humans? It has been suggested that the high rate of nondisjunction in women is a consequence of an absence or reduction in mitotic checkpoint activity during mammalian oocyte maturation (Hunt and Hassold 2002). Along these lines, it has been proposed that the prevalence of trisomies such as Down syndrome with advanced maternal age is caused by age-related reductions in the activity of mitotic checkpoint signaling members (Shonn et al. 2000). Here, our results show that meiotic chromosome segregation in vertebrates is exquisitely sensitive to a diminution in Mps1 function. Thus, even slight sex- or agedependent reductions in mitotic checkpoint signaling could permit aneuploidy in human germ cells. Interestingly, in our studies, $m p s 1^{z p 1}$ females displayed approximately twice the frequency of dysmorphic progeny and nondisjunction events as $m p s 1^{z p 1}$ males. This sex bias might be explained by a greater requirement for mitotic checkpoint activity in zebrafish oocytes, gametes that can spend hours to days arrested in meiosis I. In contrast, we saw no age-dependent effects of the $m p s 1^{z p 1}$ mutation on aneuploidy. Animals $12-14$ mo of age had a similar degree of aneuploidy as 7-10-month-old animals (K.D. Poss and M.T. Keating, unpubl.). This may reflect the fact that, whereas human oocytes are thought to spend decades arrested in meiosis I (however, see Johnson et al. 2004), zebrafish continually generate oocytes. Notably, from a group of six matings involving different 9 -month-old $m p s 1^{z p 1}$ female siblings, the percentage of dysmorphology in progeny was highly variable, ranging from $5 \%$ to $95 \%$. It is likely that this variability is due to differences in genetic background, as many genes are likely to contribute to maintaining euploidy. Pursuit of these additional genes by mutagenesis screens in zebrafish should illuminate further the etiology of aneuploidy.

\section{Materials and methods}

Mitotic checkpoint analysis

Male zebrafish from mps $1^{z p 1} /+$ matings were phenotyped at $3 \mathrm{mo}$ of age at $33^{\circ} \mathrm{C}$ for defects in fin regeneration, and used at 9 mo of age for mitotic checkpoint experiments. Animals were treated in the dark with $2 \mathrm{\mu g} / \mathrm{mL}$ nocodazole or $0.1 \%$ DMSO (untreated control group) for $12 \mathrm{~h}$ at $25^{\circ} \mathrm{C}$ or $33^{\circ} \mathrm{C}$. Testes were fixed in $4 \%$ paraformaldehyde, and processed for immunofluorescence with antibodies against $\alpha$-tubulin (Sigma) and H3P (Upstate Biotechnology). All immunofluorescence and in situ hybridization experiments were performed on cryosections as described (Poss et al. 2002b). 
Analysis of centrosomes and mitotic spindles

Testes were collected from $m p s 1^{z p 1}$ and wild-type males maintained at $25^{\circ} \mathrm{C}$ or following a $12-\mathrm{h} 33^{\circ} \mathrm{C}$ incubation, and processed for immunofluorescence. To detect centrosomes of meiotic cells, we used antibodies against $\gamma$-tubulin (Sigma) and H3P. To detect mitotic spindles in germ cells with highly condensed chromatin, we used antibodies against $\alpha$-tubulin and H3P. At least 15 meiotic cells each from four $m p s 1^{z p 1}$ and four wild-type testes were assessed microscopically; images were taken with a Zeiss LSM 510 confocal microscope.

\section{Sperm analysis}

Fish were anesthetized, and sperm was squeezed into capillary tubes for transfer to ice-cold Hank's solution. Just before FACS analysis, $10 \mu \mathrm{L} / \mathrm{mL}$ of $10 \%$ Triton $\mathrm{X}-100$ and $50 \mu \mathrm{L} / \mathrm{mL}$ of $1 \mathrm{mg} / \mathrm{mL}$ of propidium iodide in $0.1 \%$ Sodium citrate were added to each sample.

FISH

Zebrafish embryos were photographed at the 6-10-somite stage before dechorionation, cell suspension, and fixation onto slides. FISH probes were produced by fluorescently labeling bacterial artificial chromosomes derived from linkage groups 9 and 17 with Cy3-dUTP or fluorogreen-11dUTP, and hybridized and counterstained with DAPI as described (Mills et al. 1999).

\section{Genotypic analysis}

DNA was isolated from parents and dysmorphic progeny of $m p s 1^{z p 1}$ (Wik strain) $\times$ wild-type (Ekkwill strain) crosses. First, parental DNA was scored for centromere-linked CA-repeat markers representing the 25 linkage groups (Shimoda et al. 1999). Markers z3412, z11696, gof12, $z 26029$, and $z 17203$, corresponding to LG11, 13, 17, 21, and 24, respectively, showed the greatest size polymorphisms between $m p s 1^{z p 1}$ and wild-type parents. In many cases, all four parental alleles were clearly distinguishable (see Fig. 4B). DNA samples from dysmorphic progeny were then genotyped for polymorphic markers, and the frequency of monosomy or trisomy was scored at each locus and combined from all $m p s 1^{2 p 1}$ paternal crosses and from all $m p s 1^{z p 1}$ maternal crosses. Among families subject to these genotyping experiments, the frequency of morbidity strongly correlated with the frequency of nondisjunction.

\section{Acknowledgments}

We thank A. Flint for help with FACs; S. Makino for help with morphologic analysis; M. Listewnik and A. Smith for help with $\mathrm{FISH}_{\text {; }}$ and S. Johnson, D. Raible, and B. Hogan for comments on the manuscript. K.D.P. thanks the Duke Institute for Genome Sciences and Policy, the Duke Comprehensive Cancer Center, and the Whitehead Foundation for support.

The publication costs of this article were defrayed in part by payment of page charges. This article must therefore be hereby marked "advertisement" in accordance with 18 USC section 1734 solely to indicate this fact.

\section{References}

Abrieu, A., Magnaghi-Jaulin, L., Kahana, J.A., Peter, M., Castro, A., Vigneron, S., Lorca, T., Cleveland, D.W., and Labbe, J.C. 2001. Mps1 is a kinetochore-associated kinase essential for the vertebrate mitotic checkpoint. Cell 106: 83-93.

Babu, J.R., Jeganathan, K.B., Baker, D.J., Wu, X., Kang-Decker, N., and van Deursen, J.M. 2003. Rael is an essential mitotic checkpoint regulator that cooperates with Bub3 to prevent chromosome missegregation. J. Cell Biol. 160: 341-353.

Cleveland, D.W., Mao, Y., and Sullivan, K.F. 2003. Centromeres and kinetochores: From epigenetics to mitotic checkpoint signaling. Cell 112: $407-421$.

Dobles, M., Liberal, V., Scott, M.L., Benezra, R., and Sorger, P.K. 2000. Chromosome missegregation and apoptosis in mice lacking the mitotic checkpoint protein Mad2. Cell 101: 635-645.

Fisk, H.A. and Winey, M. 2001. The mouse Mpslp-like kinase regulates centrosome duplication. Cell 106: 95-104.

Fisk, H.A., Mattison, C.P., and Winey, M. 2003. Human Mps1 protein kinase is required for centrosome duplication and normal mitotic progression. Proc. Natl. Acad. Sci. 100: 14875-14880.

Grier, H.J. 1981. Cellular organization of the testis and spermatogenesis in fishes. Amer. Zool. 21: 345-357.

Hassold, T. and Hunt, P. 2001. To err (meiotically) is human: The genesis of human aneuploidy. Nat. Rev. Genet. 2: 280-291.

He, X., Jones, M.H., Winey, M., and Sazer, S. 1998. Mph1, a member of the Mps1-like family of dual specificity protein kinases, is required for the spindle checkpoint in S. pombe. J. Cell Sci. 111: 1635-1647.

Hunt, P.A. and Hassold, T.J. 2002. Sex matters in meiosis. Science 296: 2181-2183.

Johnson, J., Canning, J., Kaneko, T., Pru, J.K., and Tilly, J.L. 2004. Germline stem cells and follicular renewal in the postnatal mammalian ovary. Nature 428: 145-150.

Kalitsis, P., Earle, E., Fowler, K.J., and Choo, K.H. 2000. Bub3 gene disruption in mice reveals essential mitotic spindle checkpoint function during early embryogenesis. Genes \& Dev. 14: 2277-2282.

Kitagawa, R. and Rose, A.M. 1999. Components of the spindle-assembly checkpoint are essential in Caenorhabditis elegans. Nat. Cell Biol. 1: 514-521.

Liu, S.T., Chan, G.K., Hittle, J.K., Fujii, G., Lees, E., and Yen, T.J. 2003. Human MPS1 kinase is required for mitotic arrest induced by the loss of CENP-E from kinetochores. Mol. Biol. Cell 14: 1638-1651.

Mills, W., Critcher, R., Lee, C., and Farr, C.J. 1999. Generation of an 2.4 $\mathrm{Mb}$ human $\mathrm{X}$ centromere-based minichromosome by targeted telomere-associated chromosome fragmentation in DT40. Hum. Mol. Genet. 8: 751-761.

Nicolaidis, P. and Petersen, M.B. 1998. Origin and mechanisms of nondisjunction in human autosomal trisomies. Hum. Reprod. 13: 313319 .

Poss, K.D., Nechiporuk, A., Hillam, A.M., Johnson, S.L., and Keating, M.T. 2002a. Mps1 defines a proximal blastemal proliferative compartment essential for zebrafish fin regeneration. Development 129: 5141-5149.

Poss, K.D., Wilson, L.G., and Keating, M.T. 2002b. Heart regeneration in zebrafish. Science 298: 2188-2190.

Shimoda, N., Knapik, E.W., Ziniti, J., Sim, C., Yamada, E., Kaplan, S., Jackson, D., de Sauvage, F., Jacob, H., and Fishman, M.C. 1999. Zebrafish genetic map with 2000 microsatellite markers. Genomics 58: 219-232.

Shonn, M.A., McCarroll, R., and Murray, A.W. 2000. Requirement of the spindle checkpoint for proper chromosome segregation in budding yeast meiosis. Science 289: 300-303.

Straight, P.D., Giddings Jr., T.H., and Winey, M. 2000. Mpslp regulates meiotic spindle pole body duplication in addition to having novel roles during sporulation. Mol. Biol. Cell 11: 3525-3537.

Stucke, V.M., Sillje, H.H., Arnaud, L., and Nigg, E.A. 2002. Human Mps1 kinase is required for the spindle assembly checkpoint but not for centrosome duplication. EMBO J. 21: 1723-1732.

Uchida, D., Yamashita, M., Kitano, T., and Iguchi, T. 2002. Oocyte apoptosis during the transition from ovary-like tissue to testes during sex differentiation of juvenile zebrafish. J. Exp. Biol. 205: 711-718.

Wallace, R.A. and Selman, K. 1981. Cellular and dynamic aspects of oocyte growth in teleosts. Amer. Zool. 21: 325-343.

Weiss, E. and Winey, M. 1996. The Saccharomyces cerevisiae spindle pole body duplication gene MPS1 is part of a mitotic checkpoint J. Cell Biol. 132: 111-123.

Winey, M. and Huneycutt, B.J. 2002. Centrosomes and checkpoints: The MPS1 family of kinases. Oncogene 21: 6161-6169.

Winey, M., Goetsch, L., Baum, P., and Byers, B. 1991. MPS1 and MPS2: Novel yeast genes defining distinct steps of spindle pole body duplication. J. Cell Biol. 114: 745-754. 


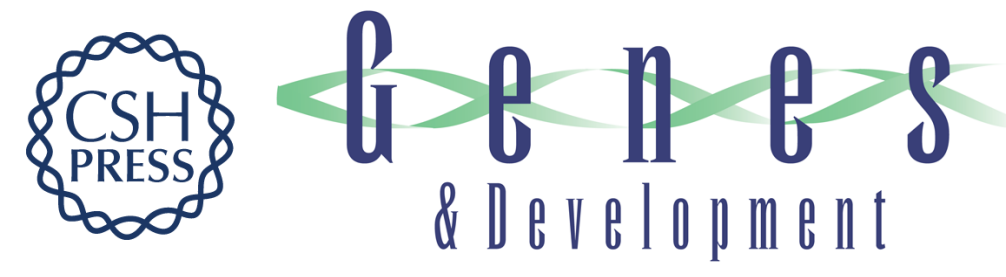

\section{Germ cell aneuploidy in zebrafish with mutations in the mitotic checkpoint gene mps1}

Kenneth D. Poss, Alex Nechiporuk, Keith F. Stringer, et al.

Genes Dev. 2004, 18:

Access the most recent version at doi:10.1101/gad.1182604

References

This article cites 25 articles, 13 of which can be accessed free at: http://genesdev.cshlp.org/content/18/13/1527.full.html\#ref-list-1

License

Email Alerting

Receive free email alerts when new articles cite this article - sign up in the box at the top Service right corner of the article or click here.

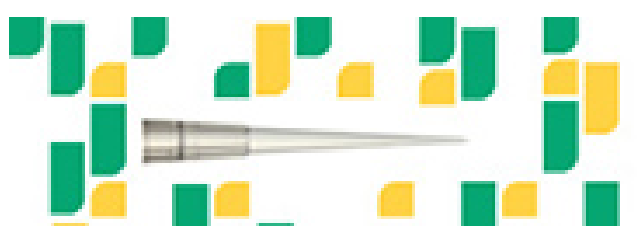

Focused on your science. 